\title{
The role of local and global properties in comparison of analogical visual scenes
}

\author{
THIERRY RIPOLL and JULIE MARTY \\ University of Provence, Aix-en-Provence, France
}

\begin{abstract}
Love, Rouder, and Wisniewski (1999) obtained interesting results showing that, in a same/different task on abstract visual scenes, subjects were able to process global properties quickly, even before local properties were identified. Our aim in this work is to explore more fully the complex relationships that exist between local processing and global processing. In our first experiment, we tested the robustness and generality of these global and local effects by using another, very different kind of local element. We showed that the global effects remain strong even when the local elements are neither conventional nor easily discriminable. In the second experiment, we showed that there exists an intermediate level of similarity between purely local and purely global similarity. Furthermore, we found that even when a stronger form of local dissimilarity is manipulated (through the introduction of different local elements), global effects were still observed. We conclude with a discussion of the respective roles of global and local properties in light of our findings.
\end{abstract}

The topic of whole/part perception has a long, controversial history with many ramifications in several domains of cognitive psychology. It is probably in the field of visual perception that this topic has been studied the most. Since the pioneering work of Navon (1977), the central question has been whether people perceive the whole first and then decompose it into parts, or perceive the parts first and then integrate them into a whole. Despite the strong interest in this question both for visual cognition and for cognition in general, many contradictory answers have been proposed (Kimchi, 1992).

In the area of visual scene comparison, Love, Rouder, and Wisniewski (1999) used an original manipulation of local/global properties and obtained very interesting results that are relevant to two classically separate domains of cognition: reasoning by analogy and visual cognition. In their experiments, subjects performed a discrimination task in which they had to indicate very quickly whether a standard matrix (i.e., a source) and a comparison matrix (i.e., a target), each composed of simple geometric elements (squares, circles, and triangles), were the same or different. The significance of this study is related to the relationship between local and global properties. In many experiments conducted to explore the local/global distinction (for example, classical nested-letters experiments), global precedence can be explained by the difference in the conspicuousness of local and global properties. However, with the material used in the present study (see Figure 1), the conspicuousness of local and global prop-

The authors thank Brad Love, Pierre Courrieu, and John Hummel for their useful comments and discussion. Correspondence concerning this article should be addressed to T. Ripoll, LPC, Université de Provence, Av. R. Schuman, 29, Aix-en-Provence 13621, Cedex 1, France (e-mail: ripoll@newsup.univ-mrs.fr). erties was controlled because it was impossible to identify global structures (i.e., to form class equivalencies between local elements) without previously identifying local elements. Consequently, any global precedence effect cannot be explained by differences in the conspicuousness of global forms. Other, more relational or structural explanations can be proposed.

Interestingly, although the subjects could respond "different" only after processing local elements (source and target matrices always differed locally on at least six local elements), they were strongly influenced by global properties. Indeed, the subjects were slower to respond "different" when the matrices were globally the same (Matrices A and B) - that is, when they were analogue. Moreover, the degrees of global and local match interacted, because local matches slowed the subjects down only when the two matrices matched globally. The latter result suggests a global precedence: When matrices were globally different, these global differences allowed the subjects to quickly respond "different" even when there were local similarities. Local similarities had a clear and strong effect only when the matrices were globally the same.

\section{Different Kinds of Local Properties and Their Influences on Global Processing}

Several crucial questions remain about the complex relationships that exist between local and global processing. These relationships are very dependent on how we define and manipulate local and global properties. The two experiments we describe here were designed to investigate how global similarity is processed when different theoretical levels of local similarity are manipulated.

Our first experiment was designed to evaluate the strength of global effect for a very different kind of local elements. In previous experiments, the local elements 
3 local matches

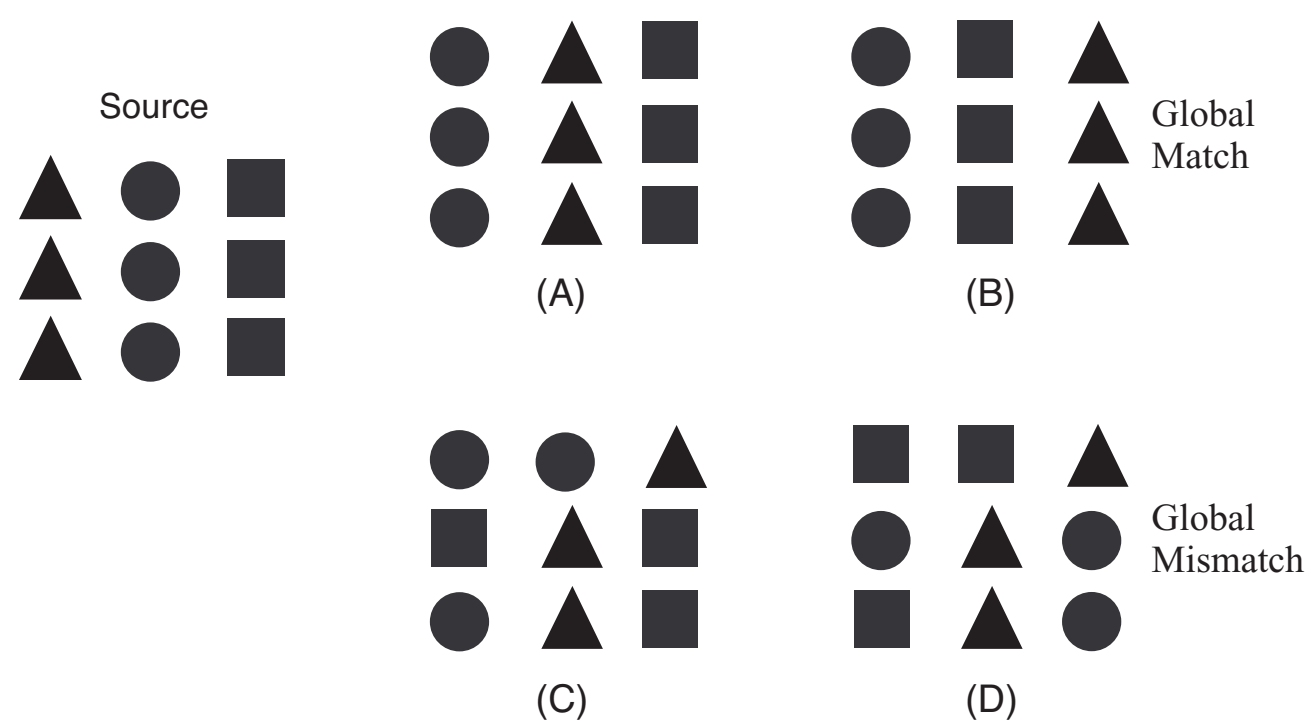

Figure 1. Stimuli used in Love, Rouder, and Wisniewski's (1999) first experiment. In the "same" condition, the target is the same as the source.

(circle, triangle, and square) had two important characteristics: They were conventional geometrical shapes that had been objects of learning in school, and they were visually very different, thereby allowing for rapid discrimination. These two characteristics can be very important because the emergence of global patterns depends on the discriminability of the local elements that make up these patterns. Global patterns are more quickly identified when local elements are easily distinguished and grouped (Duncan \& Humphreys, 1989; Nakayama \& Silverman, 1986; Treisman \& Gormican, 1988). This is theoretically important because global effects might be interpreted in two different ways: Either they result from a genuine relational processing that allows subjects to notice that the relationships between local elements are the same in two globally identical matrices, or they are a consequence of a low-level texture segregation mechanisms that allow identical elements to be grouped in a salient global pattern. In the latter case, this lowlevel texture segregation mechanism should lead less efficiently to the grouping of local elements if local elements are not easily discriminable. Consequently, and empirically, one can wonder whether the relationships between local and global processing would remain the same when the local elements do not have the characteristics described above - that is, when the local elements are neither conventional nor easily discriminable.

\section{EXPERIMENT 1}

As we stated above, the primary aim of this experiment was to evaluate relationships between global and local processing for a very different kind of local differences. In this way, it constitutes both a replication and a generalization of the results obtained by Love et al. (1999). For this reason, the experiment differed from Love et al.'s experiment only in the materials used.

\section{Method}

Subjects. The 18 subjects were first- and second-year psychology students at the University of Provence.

Apparatus. The experiment was run on Macintosh computers with 12-in. color monitors.

Stimuli. The stimuli were $3 \times 3$ matrices, each consisting of three kinds of abstract, unfamiliar forms (see Figure 2). Six different versions of each pattern were constructed by rearranging the local elements while preserving the global pattern. All the matrices had a salient global pattern. The sizes of the matrices were the same as those in Love et al.'s (1999) experiments.

Design. The two factors - global similarity and local matcheswere manipulated orthogonally, and each subject underwent all four experimental conditions. Examples of the four different conditions are illustrated in Figure 2. Each subject participated in 144 trials, 48 of which were "same" trials, in which the target was the same as the source. The remaining 96 trials were "different" trials, with 24 trials for each of the four source-target mismatch conditions. The order of the trials was randomized for each subject.

Procedure. The same procedure was used as in Love et al.'s (1999) experiments. Very generally, the subjects were seated $40 \mathrm{~cm}$ from the computer monitor. The source was presented for $1,000 \mathrm{msec}$ on the left half of the screen. Then, 1,000 msec after the onset of the source, a frame with a fixation cross was displayed on the right half of the screen for $500 \mathrm{msec}$. After $500 \mathrm{msec}$, the source and the frame disappeared and the target was displayed inside the previous position of the frame. The target remained on the screen until the subjects responded.

\section{Results}

The subjects responded correctly on $87 \%$ of the "same" trials and on $90 \%$ of the "different" trials. This percentage differed slightly among the four "different" conditions, but not significantly (see Table 1). However, the error rates in the slower response conditions tended to be higher. 


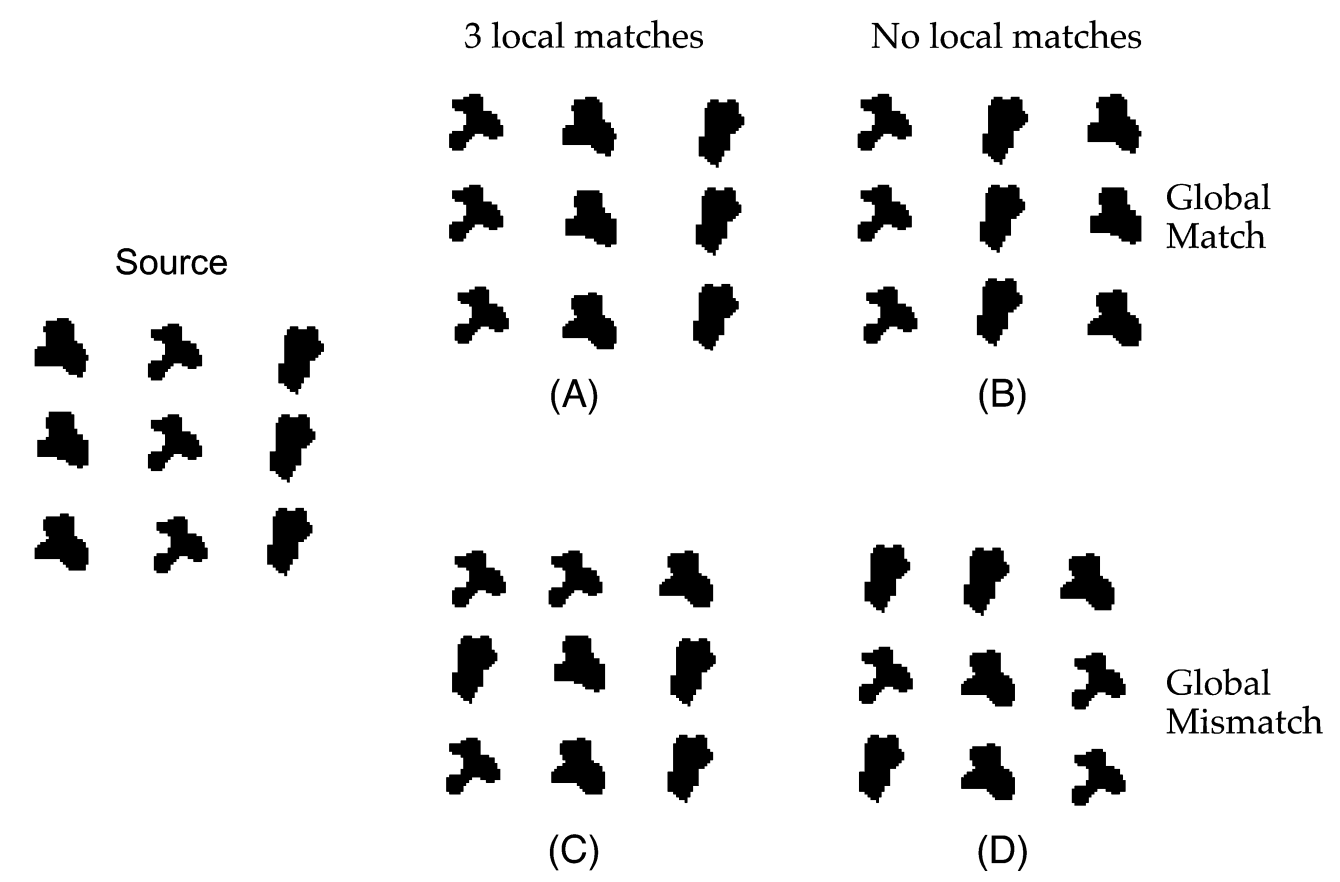

Figure 2. Stimuli used in Experiment 1. In the "same" condition, the target is the same as the source.

For the response time (RT) analysis, we discarded data only if the RT was less than $200 \mathrm{msec}$ (these discarded trials comprised less than $1 \%$ of the total). Because we were interested mainly in the "different" trials, only the results relative to the four "different" conditions ${ }^{1}$ will be discussed. Because error frequency was not the same across the four "different" conditions, we performed two analyses of variance (ANOVAs): one that excluded RTs associated with error data and one that included them. These analyses revealed exactly the same pattern of results, so we will present only the response time analysis for correct responses.

Table 1 shows the mean correct RTs for the "different" trials. A mean correct RT was calculated for each subject in each condition, and a 2 (global similarity: global match vs. global mismatch) $\times 2$ (local matches: three local matches vs. zero local matches) repeated measures ANOVA was performed, with mean RT as the dependent variable.

The subjects were slower (1,131 vs. $979 \mathrm{msec})$ to respond "different" for trials in which the source and the target were globally the same than when they were glob-

Table 1

Mean Correct Response Times (RTs, in Milliseconds) and Correct Response Percentages (\%Correct) for the "Different" Trials in Experiment 1

\begin{tabular}{lccccc}
\hline & \multicolumn{2}{c}{ Three Local Matches } & & \multicolumn{2}{c}{ No Local Matches } \\
\cline { 2 - 3 } \cline { 5 - 6 } \multicolumn{1}{c}{ Condition } & RT & \%Correct & & RT & \%Correct \\
\hline Global match & 1,220 & 85 & & 1,042 & 89 \\
Global mismatch & 982 & 92 & & 975 & 95 \\
\hline
\end{tabular}

ally different $\left[F(1,17)=22.141, M S_{\mathrm{e}}=18,806.824, p<\right.$ $.001]$. They were also slower $(1,101 \mathrm{vs} .1,009 \mathrm{msec})$ to respond "different" when there were three local matches than when there were no local matches $[F(1,17)=22.257$, $\left.M S_{\mathrm{e}}=6,809.536, p<.001\right]$. We also found a strong interaction between the two factors $[F(1,17)=18.593$, $\left.M S_{\mathrm{e}}=7,111.933, p<.001\right]$. The effect of local matches was stronger in the global match condition than in the global mismatch condition.

\section{Discussion}

As a whole, we found the same pattern of results as in Love et al. (1999). The only differences concerned the RTs, which were longer in this experiment. Although the subjects took more time to answer, they experienced the same difficulty discriminating the matrices that were both globally and locally similar. The most interesting finding is that we reproduced the same pattern of results with very different materials, which suggests that the phenomenon that Love et al. discovered is robust and general. Even when local elements are not conventional elements and even when their distinctiveness is not great, global structure seems to be processed before local elements are identified.

\section{EXPERIMENT 2}

The first goal of this experiment was to clearly distinguish two very different kinds of local similarities/ differences that were not previously identified. Indeed, two visual stimuli can differ locally in two ways: They can differ either because the same local elements are not 
located at the same position, or because the local elements are simply different. In previous experiments, local differences were always of the first type. In this case, to solve the task, local processing required subjects to simultaneously process both the nature and the location of the local elements. Of course, this kind of local processing must be quite costly because it implies taking into account two pieces of information that the perceptual system processes differently (Lavie \& Driver, 1996; Vecera \& Farah, 1997). Many studies on visual attention have clearly shown that locating an object is more demanding than simply identifying it (van der Heijden, 1992). Furthermore, such complex information can hardly be stored in visual short-term memory, whose capacity is limited to four units (Vogel, Woodman, \& Luck, 2001). On the contrary, as was suggested by these previous attentional and visual memory studies, when the nature of local elements is involved in local differences, a more primitive and less costly way of processing should allow very rapid and effortless detection of local differences. It seems probable that one can more rapidly determine whether a local target element was present/absent in the source than whether a given element was in the same place in both the source and the target. Thus, one may wonder if early global effects still occur when local processing involves only the nature of the local elementsfor example, in situations in which one or more local element that is present in the target is absent from the source (see Figure 3). If global effects persist for this kind of local processing, then this would provide a stronger demonstration that global properties can be identified and used very early during the comparison of visual scenes.
The other goal of this experiment relates to the way in which local similarity has been manipulated previously. Indeed, local matches did not have the same status in global match and global mismatch conditions. The local matches in the global match condition (see Figures 1 and 2) necessarily were related to three contiguous elements that were all the same (squares), whereas in the global mismatch condition the local matches were related to two different, noncontiguous elements (two squares and a circle). Due to formal constraints, the local matches in the global match condition were a part of the source's global pattern (a column of like elements), whereas this was not the case in the global mismatch condition. Consequently, these two kinds of local matches are theoretically and conceptually very different. In a way, the local matches manipulated in the global match condition were not totally local, because they were part of the global pattern. From a theoretical point of view, they correspond to an intermediate level of similarity between purely local (involving a single element) and purely global (involving the whole configuration) similarity.

One of the consequences of this analysis is that the interaction we obtained previously can be interpreted in two very different ways. The first interpretation is that this interaction reveals a clear global precedence. The second interpretation is that local match effects in the global match condition were stronger because local elements formed an intermediate level between local and global similarities. To resolve this ambiguity, local similarity in global match and global mismatch conditions should be perfectly comparable. However, this goal is very difficult to reach because it is formally impossible

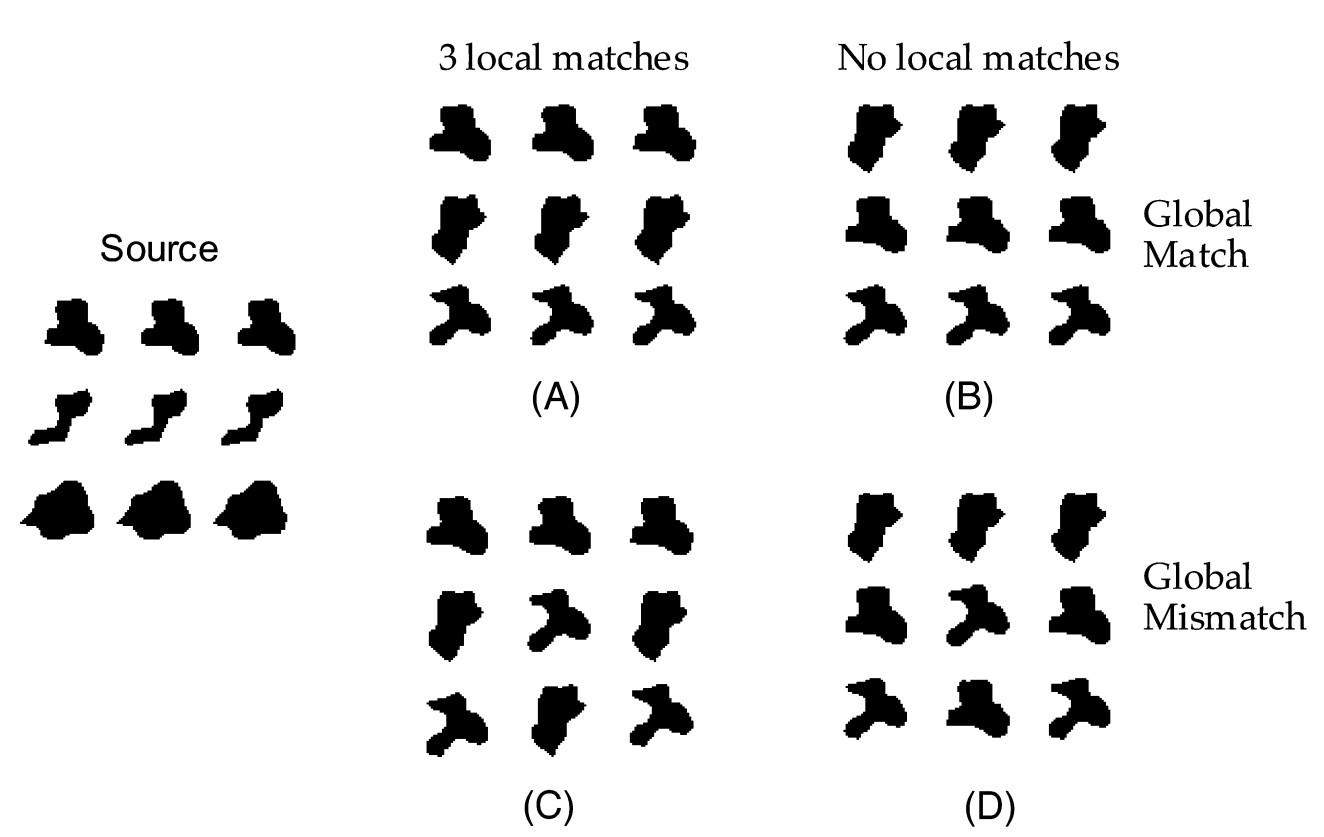

Figure 3. Stimuli used in Experiment 2. In the "same" condition, the target is the same as the source. 
to manipulate information at one level (e.g., local or global) without affecting the other. This phenomenon results from the fact that the global pattern is defined by the positions of the local elements: Local and global properties are interdependent. For example, with the material presented in Figure 1, one cannot introduce local matches on different elements in Matrix A without changing its global organization. The local and global levels are too dependent and too closely linked to allow for orthogonal manipulation. Consequently, one can solve this problem only by modifying the constraints implicitly adopted to generate the matrices. One solution consists in introducing new local elements into the target (i.e., local target elements different from the local source elements). Thus, there are more degrees of freedom for generating the matrices and it becomes possible to give the same properties to the local match elements in the global match (Matrix A) and global mismatch (Matrix C) conditions. For example, by using the same elements in the source and in the target, it would be impossible, with the global pattern of Matrix C (Figure 3), to have no more matches than the three local matches in the top row. Unless new local elements are used in the target, the number of local matches will exceed three because, whatever the global pattern, there will necessarily be at least two other local matches in the bottom two rows. By putting new elements in the target, it is possible to create the global pattern we need in order to have no more than three local matches and to preserve the same status for the local matches in the global match and global mismatch conditions. As is shown in Figure 3, the three local matches in the global match and global mismatch conditions are the same (three contiguous identical elements).

With this control, any different local match effects in the global match and global mismatch conditions (interaction) should be interpreted as a manifestation of global precedence. By contrast, the absence of an interaction would suggest that there is an intermediate level of similarity between purely local and purely global similarities. Finally, the introduction of new elements into the target allows us to evaluate the strength of global processing when local elements differ in nature.

\section{Method}

Subjects. The 20 subjects were first- and second-year psychology students at the University of Provence.

Apparatus. The experiment was run on Macintosh computers with 12-in. color monitors.

Stimuli. The only difference from the previous experiment was that two new forms were introduced into the set of stimulus matrices when the source and target were different (see Figure 3). Note that local matches could appear at any place in the matrix (top, bottom, left, right, or center position) and could be organized in columns, rows, or any other kind of more complex form. In this way, the subjects could not predict from the visual information available in the source anything about what information would be diagnostic or nondiagnostic in the target.

Design. The design was the same as in the previous experiment. The four conditions are illustrated in Figure 3. Each subject participated in 160 trials, of which 32 were "same" trials. The remaining
128 trials were "different" trials, with 32 trials for each of the four mismatch conditions. The order of the trials was randomized for each subject.

For methodological reasons, two kinds of filler conditions were introduced. The first involved the addition of 64 pairs of identical matrices (with patterns different from those used in the experimental conditions). This was used to balance the numbers of "same" and "different" trials. The second involved the addition of 64 pairs of matrices in which the source and the target were made up of the same elements but were nevertheless different (see Figure 4). This filler condition was necessary to prevent the subjects from adopting a strategy in which they would systematically respond "same" when the source and target elements were the same and "different" when the source and target elements were different. It is important to mention that the subjects could not discriminate fillers from others pairs of matrices. ${ }^{2}$ The subjects saw a total of 288 pairs of matrices (160 experimental pairs and 128 filler pairs).

Procedure. The same procedure was used as in the previous experiment.

\section{Results}

Trials were discarded only if the RT was less than $200 \mathrm{msec}$ (these discarded trials comprised less than $1 \%$ of the total). The subjects responded correctly on $90 \%$ of the "same" trials and on $91 \%$ of the "different" trials. This percentage differed slightly among the four "different" conditions, but not significantly (see Table 2). However, the error rate in the slower response conditions tended to be higher. Given that we are interested mainly in the "different" trials, only the results relative to the four "different" conditions will be discussed.

Table 2 shows the mean correct RTs for the "different" trials. A mean correct RT was calculated for each subject in each condition, and a 2 (global similarity: global match vs. global mismatch) $\times 2$ (local matches: three local matches vs. zero local matches) repeated measures ANOVA was performed, with mean RT as the dependent variable.

The subjects were slower $(1,166 \mathrm{vs} .959 \mathrm{msec})$ to respond "different" for trials in which the source and the target were globally the same than when they were globally different $\left[F(1,19)=65.158, M S_{\mathrm{e}}=13,204.387, p<\right.$ $.0001]$. They were also slower $(1,116 \mathrm{vs} .1,009 \mathrm{msec})$ to respond "different" when there were three local matches than when there were no local matches $[F(1,19)=30.016$, $\left.M S_{\mathrm{e}}=7,555.782, p<.0001\right]$. However, there was no interaction between the two factors $[F(1,19)=0.013$, $\left.M S_{\mathrm{e}}=13,818.459, p=.91\right]$. Each factor had a strictly

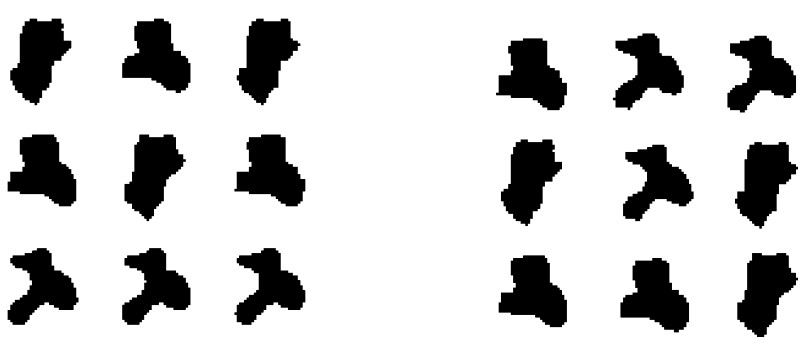

Figure 4. A pair of filler matrices. 
Table 2

Mean Correct Response Times (RTs, in Milliseconds) and Correct Response Percentages (\%Correct) for the "Different" Trials in Experiment 2

\begin{tabular}{lccccc}
\hline & \multicolumn{2}{c}{ Three Local Matches } & & \multicolumn{2}{c}{ No Local Matches } \\
\cline { 2 - 3 } \cline { 5 - 6 } \multicolumn{1}{c}{ Condition } & RT & \%Correct & & RT & \%Correct \\
\hline Global match & 1,221 & 87 & & 1,111 & 92 \\
Global mismatch & 1,011 & 90 & & 907 & 95 \\
\hline
\end{tabular}

additive effect, and the effect of local matches was the same in the global match and global mismatch conditions.

\section{GENERAL DISCUSSION}

The first experiment of this study clearly shows that previously obtained global effects were not limited to a very special class of stimuli. The strong global effect and the interaction were replicated on a very different class of stimuli: nonconventional, poorly discriminable stimuli. These initial results are theoretically important, but they are also methodologically useful because they allow for an accurate evaluation of the local/global effects we observed in the second experiment.

The first interesting result of the second experiment is that global effects were always observed, even in a situation in which the two matrices differed by the introduction of new elements in the target. Recall that six of the nine target elements were new. In this case, the cost of processing local similarity could be very low because the nature of the new local elements (and not their position) was sufficient to allow quick, low-risk resolution of the task. Nevertheless, even with this stronger manifestation of local dissimilarity, the subjects were slowed down by global similarity. Other experiments would be necessary to evaluate the generality of these results. In particular, one can wonder whether global effects would remain if different local elements in the target were more discriminable and conventional.

The second interesting point is related to the disappearance of the interaction. Even if one advocates an opportunistic theoretical approach in which the order of processing (global first or local first) depends on the salience of information pertinent to each mechanism, the interaction found in previous experiments is strongly consistent with the fact that global correspondences are made before local correspondences. However, as we hypothesized before, this interaction could be a consequence of the different status of local matches in global match and global mismatch conditions. By causing the disappearance of the interaction, the methodological control we introduced allows us to draw two important conclusions. First, these results are consistent with a strong parallel hypothesis, because if global properties were processed before local properties we would have found the interaction of our first experiment. Local and global properties seem to have been processed simulta- neously without any local or global precedence. Second, it seems that there is an intermediate level of similarity between a purely local and a wholly global level of similarity. This level of similarity corresponds to a part of the global pattern. It seems very probable that the previous interaction was a consequence of the fact that what we called a local similarity in global match and global mismatch conditions actually corresponded to two different kinds of similarity. Any future attempt to study relationships between local and global similarities should take into account the existence of these different levels of similarity.

\section{Implications for Analogical Models of Reasoning by Analogy}

Because the explanation of local and global processing is structural rather than strictly perceptual, and because this material highlights the importance of structural principles in determining global correspondences, these results have certain implications for theories of structural comparison in higher level cognition (e.g., analogy). They suggest a more flexible approach (see, e.g., Hummel \& Holyoak, 1997) than the strictly localto-global ones. However, we must acknowledge that the theoretical transfer from perceptual tasks to conceptual tasks is not so straightforward, even if there is general agreement that high-level cognition is strongly connected to perceptual cognition. In fact, the main difficulty encountered in transferring this more flexible approach to the domain of analogy and conceptual similarity is not linked to the characteristics of similarity processing per se. It would be very easy for models such as ACME, SIAM, or LISA, which are highly parallel models, to simultaneously process global and local properties. The main problems lie in defining what a global property is and determining how subjects detect global properties when stimuli are more conceptual than perceptual. In previous studies on reasoning by analogy (Ripoll, 1998; Ripoll, Brude, \& Coulon, 2003; Ripoll \& Coulon, 2002), we showed that analogical retrieval, like analogical transfer, was possible before subjects aligned the two structures and before they mapped the local properties of the two analogues (for a similar approach, see also Ross \& Bradshaw, 1994; Schunn \& Dunbar, 1996; Wisniewski $\&$ Bassok, 1999). In this case, subjects used global properties inferred from the source to process the target. Such processing of analogies or conceptual comparisons is very useful because it substantially lightens the load and can have great heuristic value. However, although it is rather easy to manipulate these global properties in a particular experimental context, it is much more difficult to formally and generally define what these global properties are for linguistic stimuli. Nevertheless, converging data from visual and conceptual tasks demonstrating the importance of global processing should encourage researchers to define these properties more rigorously and formally, in view of introducing them into current models of reasoning by analogy. 


\section{REFERENCES}

Duncan, J., \& Humphreys, G. W. (1989). Visual search and stimulus similarity. Psychological Review, 96, 433-458.

Hummel, J. E., \& HolyOAK, K. J. (1997). Distributed representations of structure: A theory of analogical access and mapping. Psychological Review, 104, 427-466.

KIMCHI, R. (1992). Primacy of wholistic processing and global/local paradigm: A critical review. Psychological Bulletin, 112, 24-38.

Lavie, N., \& Driver, J. (1996). On the spatial extent of attention in object-based visual selection. Perception \& Psychophysics, 58, 12381251.

Love, C. L., Rouder, J. N., \& Wisniewski, E. J. (1999). A structural account of global and local processing. Cognitive Psychology, 28, 291-316.

NaKayama, K., \& Silverman, G. H. (1986). Serial and parallel processing of visual feature conjunctions. Nature, 320, 264-265.

Navon, D. (1977). Forest before the trees: The precedence of global features in visual perception. Cognitive Psychology, 9, 353-383.

Ripoll, T. (1998). Why this makes me think of that. Thinking \& Reasoning, 4, 15-43.

RiPOLL, T., BRUDE, T., \& Coulon, D. (2003). Does analogical transfer involve a term-to-term alignment? Memory \& Cognition, 31, 221230.

Ripoll, T., \& Coulon, D. (2002). Encodage analogique et transfert sans mapping [Is mapping a necessary condition for analogical transfer?]. L'Anée Psychologique, 102, 619-656.

Ross, B. H., \& Bradshaw, G. L. (1994). Encoding effects of remindings. Memory \& Cognition, 22, 591-605.
SchunN, C. D., \& Dunbar, K. (1996). Priming, analogy, and awareness in complex reasoning. Memory \& Cognition, 24, 271-284.

Treisman, A., \& GormicAN, S. (1988). Feature analysis in early vision: Evidence from search asymmetries. Psychological Review, 95, 1548.

VAN DER HeIJDEN, A. H. C. (1992). Selective attention in vision. London: Routledge.

Vecera, S. P., \& Farah, M. J. (1997). Is visual image segmentation a bottom-up or an interactive process? Perception \& Psychophysics, 59, 1280-1296.

VoGEL, E. K., Woodman, G. F., \& Luck, S. J. (2001). Storage of features, conjunctions, and objects in visual working memory. Journal of Experimental Psychology: Human Perception \& Performance, 27, 92-114.

WISNIEWSKI, E. J., \& BASSOK, M. (1999). What makes a man similar to a tie? Stimulus compatibility with comparison and integration. $C_{0}$ nitive Psychology, 39, 208-238.

\section{NOTES}

1. The results of the "same" condition were consistent with Love et al.'s (1999) results (the mean RT was 1,310 msec in this experiment).

2. Two preliminary experiments were conducted without fillers. The same pattern of results was obtained, but global effects were slightly weaker, probably because the subjects paid more attention to local elements.

(Manuscript received June 13, 2002;

revision accepted for publication April 8, 2004.) 